\title{
Multi-scale processes in metapopulations: contributions of stage structure, rescue effect, and correlated extinctions
}

\author{
Chris Sutherland, ${ }^{1,2}$ David A. Elston, ${ }^{2}$ and Xavier Lambin ${ }^{1,3}$ \\ ${ }^{1}$ School of Biological Sciences, University of Aberdeen, Aberdeen, United Kingdom \\ ${ }^{2}$ Biomathematics and Statistics Scotland, Craigiebuckler, Aberdeen, United Kingdom
}

\begin{abstract}
Metapopulations function and persist through a combination of processes acting at a variety of spatial scales. Although the contributions of stage structure, spatially correlated processes, and the rescue effect to metapopulation dynamics have been investigated in isolation, there is no empirical demonstration of all of these processes shaping dynamics in a single system. Dispersal and settlement differ according to the life stage involved; therefore, stage-specific population size may outperform total population size when predicting colonization-extinction dynamics. Synchrony in patch dynamics can lead to accelerated metapopulation extinction, although empirical evidence of the interplay between correlated colonization events and correlated extinctions is lacking. Likewise, few empirical examples exist that provide compelling evidence of migration acting to reduce extinction risk (the rescue effect). We parameterized a hierarchy of metapopulation models to investigate these predictions using a seven-year study of a naturally occurring water vole (Arvicola amphibius) metapopulation. Specifically, we demonstrated the importance of local stage structure in predicting both colonization and extinction events using juvenile and adult population sizes, respectively. Using a novel approach for quantifying correlation in extinction events, we compared the scale of synchrony in colonization and extinction. Strikingly, the scale of dispersal acting to synchronize colonization was an order of magnitude larger than that of correlated extinctions (halving distance of the effect: $12.40 \mathrm{~km}$ and $0.89 \mathrm{~km}$, respectively). Additionally, we found compelling evidence for the existence of a nontrivial rescue effect. Here we provide a novel empirical demonstration of a variety of metapopulation processes operating at multiple spatial scales, further emphasizing the need to consider stage structure and local synchrony in the dynamics of spatially dependent, stage-structured (meta) populations.
\end{abstract}

Key words: Arvicola amphibius; Bayesian models; colonization; dispersal; metapopulation; rescue effect; scale; spatially correlated extinction; stage structure; stochastic patch occupancy model, SPOM; water vole.

\section{INTRODUCTION}

Considering the effects of spatial structure and habitat characteristics has improved the way we understand the dynamics of fragmented populations (Hanski and Gaggiotti 2004). An important generality emerging from spatially realistic metapopulation theory is the area-isolation paradigm, which states that colonizationextinction dynamics can be reasonably described using patch size and the proximity to extant populations (Hanski 1994, 1998, Moilanen 1999, Ovaskainen and Hanski 2004). Spatially realistic stochastic patch occupancy models, SPOM (Hanski 1994, Day and Possingham 1995, Moilanen 1999, Hanski and Ovaskainen 2000, Ovaskainen and Hanski 2004) are a class of models that incorporate this simple yet realistic description of the landscape, and are well suited to investigate the area-isolation relationship (Etienne et al. 2004). This

Manuscript received 1 February 2012; revised 3 May 2012; accepted 10 May 2012. Corresponding Editor: M. K. Oli.

${ }^{3}$ Corresponding author. E-mail: x.lambin@abdn.ac.uk paradigm is well understood and is supported in a range of natural settings (e.g., see review by Pellet et al. 2007). Theoretical, experimental, and to a lesser extent empirical, studies, however, have investigated a range of additional processes that, in isolation, can shape metapopulation dynamics (e.g., local dynamics, correlated processes, and the rescue effect). An enduring challenge is to determine whether multi-scale processes operate in real metapopulations.

Connectivity is a fundamental concept in metapopulation ecology that describes the rate of migration to, or from, a local population or habitat patch (Moilanen and Nieminen 2002, Prugh 2009). Measures of connectivity that consider distance to all extant populations, weighted by their size (area), outperform nearest neighbor or buffer distances when predicting colonization (Moilanen and Nieminen 2002). Moreover, the use of patch size reflects an assumption that population size is proportional to patch area (Hanski 1994, Ovaskainen and Hanski 2004) and that larger patches send out more dispersers. This relationship extends also to extinction, 
such that smaller patches are more likely to go extinct than larger ones (Hanski 1994, Ovaskainen and Hanski 2004).

The use of patch size allows for convenient simplifying assumptions (Hanski 1994, Ovaskainen 2002, Moilanen 2004, Ovaskainen and Hanski 2004). However, empirical evidence indicates that extinction events are predicted more effectively by (time-varying) population size than by time-invariant patch size, affirming the role of demography in the extinction process (Pellet et al. 2007). Direct comparisons between neighborhood patch size and population size to measure connectivity, however, are less common. Density-dependent dispersal (Matthysen 2005), Allee effects (Gyllenberg et al. 1999) or conspecific attraction and avoidance (Lehmann and Perrin 2003) suggest that incorporating population size and structure may be more informative than patch size when used to explain colonization-extinction dynamics. Ozgul et al. (2009) provide a compelling empirical demonstration of the differential effects of stage structure on long- and short-term metapopulation dynamics (but see also Crone et al. 2001, Clinchy et al. 2002, Baguette and Schtickzelle 2003, Schooley and Wiens 2005). Here we note that, although it constrains the predictive capabilities of SPOMs to one-step-ahead predictions of occupancy (Ovaskainen 2002), investigating the roles of population size and structure is an important step in understanding determinants of metapopulation functioning (Hanski and Gaggiotti 2004).

In reality, the dynamics of spatially structured populations are subject to a range of potentially synchronizing processes operating at a variety of spatial scales (Holt 1993, Heino et al. 1997, Bjørnstad et al. 1999, Koenig 1999, Liebhold et al. 2004). Synchrony of local populations can arise through regional stochasticity (e.g., climatic/weather conditions, predation, disease, and habitat loss/change) or the metapopulation dynamics themselves, and an understanding of the scale of correlated dynamics can prove useful when inferring the underlying biological processes involved (Hanski 1991, Heino et al. 1997, Moilanen 1999, Clinchy et al. 2002, Ovaskainen 2002, Ovaskainen and Hanski 2003, Kallimanis et al. 2005). Because dispersal introduces spatial synchrony between populations, colonization is spatially correlated (Bjørnstad et al. 1999, Koenig 1999, Liebhold et al. 2004) and the scale of the correlation is determined by the (estimated) dispersal kernel (Hanski 1994, 1997). Spatially correlated extinctions can reduce the time to metapopulation extinction by limiting the capacity and the effective number of patches in the system (Moilanen 1999, Ovaskainen 2002, Ovaskainen and Hanski 2003, Kallimanis et al. 2005). Metapopulations can be buffered from such detrimental effects if dispersal distances are relatively large, and can therefore persist despite spatially correlated extinctions (Heino et al. 1997, Ovaskainen et al. 2002, Lambin et al. 2004). Measuring the scale of spatial correlation in real metapopulations remains an important area of research.
Dispersal-induced synchrony can also result in populations in dense neighborhoods having reduced probabilities of extinction, known as a rescue effect (Brown and Kodric-Brown 1977). When a rescue effect is present, a degree of spatial correlation is introduced into population persistence. Although it is mathematically interesting, empirical evidence for the rescue effect is rare and not uncontroversial (Moilanen et al. 1998, Etienne 2000, Clinchy et al. 2002).

We present a retrospective analysis of seven years of patch occupancy data from a metapopulation of water voles, Arvicola amphibius, in which we parameterize and statistically fit a hierarchy of SPOMs to patch occupancy histories and their associated population size. We directly investigate the presence (hitherto lacking in the empirical literature) of multiple processes operating at different spatial scales in a single, naturally occurring metapopulation. First, we relax the assumption that patch size is a true reflection of the effective population size. Using within-patch population size and structure, we investigate the relative contributions of different life stages to colonization-extinction dynamics. We then extend the concept of connectivity, traditionally used to characterize the scale of dispersal, to estimate the scale of spatially correlated extinction. We can therefore compare the spatial scale of both processes and assess their effect on metapopulation functioning. Finally, our approach, applied to a natural metapopulation, allows us to provide rare and compelling empirical support for the existence and influence of a rescue effect.

\section{Methods}

\section{Assynt water vole data}

The study area is a heather-dominated upland landscape $\sim 140 \mathrm{~km}^{2}$ in size and is located in the Assynt area, northwestern Scotland (see also Appendix B). In Assynt, water voles can grow up to $300 \mathrm{~g}$, live no more than two years, and occupy discrete vegetated stretches of riparian habitat (patches, hereafter) that are embedded within the unsuitable heather matrix. Water vole patches are, on average, $0.847 \mathrm{~km}$ long (range: 0.067$3.007 \mathrm{~km}$ ) and make up around $11 \%$ of the $860-\mathrm{km}$ waterway network in the study area. In the year of birth, water voles disperse from their natal patch and a small fraction may mature sexually. In their second year, females hold a territory, produce 1-3 litters, and most adults $(>99 \%)$ are never seen again. We therefore consider this water vole metapopulation to have nonoverlapping breeding generations; that is, most adults perish before their offspring reproduce. In 1999, the study area was comprehensively surveyed and all suitable water vole habitat patches were mapped. In this analysis, we consider data from 69 suitable water vole habitat patches collected during the breeding period (July and August) of each year from 1999 to 2005. The data consist of (1) patch occupancy states for each patch in each year and, (2) population size and structure of each of the occupied patches in each year. 
To generate patch occupancy histories, each of the 69 sites was visited only once in each year and intensively surveyed for the presence or absence of fresh water vole signs, mainly latrines (piles of feces used for territory marking). When signs were detected, patches were scored as occupied (1); otherwise they were scored as empty (0), providing what is commonly referred to as snapshot data. At all patches that were observed as being occupied, trapping was carried out over 3-5 consecutive days and, when captured, water voles were marked, aged (juvenile or adult), and sexed (for more detail, see Aars et al. 2001). The median number of water voles in a colony was 2 adults (range: $1-16$ ) and 3 juveniles (range: 1-22). Using closed-capture models (program MARK; White and Burnham 1999), the estimated probability of a water vole being captured at least once over four days of trapping was high (cumulative probability that a vole is caught after 4 days of trapping: 0.94 [95\% bootstrapped confidence interval $0.92-0.95]$ for adults and 0.94 [95\% bootstrapped confidence interval 0.93-0.96] for juveniles). The data did not allow for patch-within-year-level estimates of trappability, although pooling across patches within years showed no significant between-year differences in the probability that an individual is captured at least once (for further details on estimating trappability, see Appendix A). Therefore, given that colony sizes were small and that the probability of being captured over 4 days was very high, we considered the live-trapping data to be a representative estimate of the true local population size and structure.

\section{The statistical model}

A SPOM describes the transitions of $n$ discrete habitat patches between two occupancy states: occupied $(P=1)$ and empty $(P=0)$. It is defined as a first-order Markov chain with $2^{n}$ possible states in which the metapopulation state at time $t+1$ depends on the state at time $t$ (reviewed and discussed in Etienne et al. 2004, Moilanen 2004, Ovaskainen and Hanski 2004). Patch transition probabilities are modeled using two submodels that describe the processes of colonization $(C)$ and extinction $(E)$. The colonization probability of an empty patch in a given year is assumed to be caused by immigrating individuals and is modeled as an asymptotically increasing function of spatial connectivity to extant patches in the previous year: $C_{i, t}=1-\exp \left(-S_{i, t}\right)$ (Hanski 1997, Moilanen 2004). Connectivity, $S_{i, t}$, is therefore the component that defines colonization. Connectivity describes the distance-dependent influence of all potential neighboring source populations via a negative exponential dispersal kernel (Hanski 1994, Moilanen and Nieminen 2002):

$$
S_{i, t}=\gamma \sum_{j \neq i} A_{j} P_{j, t-1} \exp \left(-\alpha d_{i, j}\right)
$$

Here, $P_{j, t-1}$ is the occupancy state of the $j$ th neighboring site at time $t-1 ; A_{j}$ is the corresponding time-invariant patch size; and $d_{i, j}$ is the Euclidean distance (here, in kilometers) between patch $i$ and $j$. The term $\exp \left(-\alpha d_{i, j}\right)$ describes the dispersal kernel with the scaling parameter $\alpha$, which can be related to the halving distance of colonization pressure from neighboring extant patches by $\log (2) / \alpha$. Parameter $\gamma$ is the population-level per capita effective dispersal rate.

The probability of a population going extinct is typically defined as a decreasing function of the patch size. Here the extinction probability is defined as $\operatorname{logit}\left(E_{i, t}\right)=\beta_{0}+\beta_{1} A_{i}$, where $A_{i}$ is the time invariant patch size, $\beta_{0}$ is the intercept, and $\beta_{1}$ is the slope that relates extinction probability to patch size (or population size). A rescue effect can be incorporated into the SPOM framework by adjusting the extinction probability (to $E_{i, t}^{*}$ ) to allow for a decreasing influence of migration on extinction probabilities: $E_{i, t}^{*}=\left(1-C_{i, t}\right) E_{i, t}$ (Moilanen 2004).

A major benefit of using SPOMs is that they provide a flexible framework within which a range of biological hypotheses can be tested using competing functional relationships to characterize the colonization and extinction processes (Hanski and Gaggiotti 2004). It is also important to note that, although a suite of modern tools exists to model site occupancy dynamics, these require multiple within-season site visits resembling a robust design (occupancy models; MacKenzie et al. 2003). The SPOM framework is therefore more suited to answer interesting questions about factors influencing colonization-extinction dynamics in situations where only single-visit, snapshot data are available; as is the case in this study.

\section{Stage structure and correlated extinctions: alternative parameterizations}

First, we relaxed the assumption that patch size is a true reflection of the effective population size and investigated whether within-patch population size and structure may be a better predictor of colonizationextinction dynamics than patch size. To do this we considered three alternative population sizes as covariates and substitutes for patch size: juvenile only, adult only, and total population (collectively, $P_{i, t}^{*}$, hereafter). Stage structure effects on colonization were investigated by substituting the patch size and occupancy state term, $A_{j} P_{j, t-1}$, in connectivity (Eq. 1) with the number of individuals of a given stage present in time $t-1, P_{j, t-1}^{*}$. In the same way, the effects of stage structure on extinction were investigated by substituting $A_{i}$ in the extinction term with $P_{i, t}^{*}$.

Using connectivity to capture spatial structure in colonization is common in studies of metapopulation dynamics, but empirical evidence of spatially correlated extinction is less common, as is the assessment of how synchrony in both processes drives metapopulation dynamics in nature. To account for spatially correlated extinctions, we extended the extinction function to include a connectivity variable, $S_{i, t}^{e}$, similar to that in 
TABLE 1. DIC (deviance information criteria) values used to compare alternative parameterizations of a SPOM (stochastic patch occupancy model) for colonization and extinction by life stage.

\begin{tabular}{|c|c|c|c|c|c|c|}
\hline \multirow[b]{2}{*}{ Colonization } & \multirow[b]{2}{*}{ Extinction } & \multicolumn{2}{|c|}{ No SCE } & \multicolumn{2}{|c|}{ SCE } & \multirow[b]{2}{*}{$\Delta \mathrm{DIC}$} \\
\hline & & No rescue effect & Rescue effect & No rescue effect & Rescue effect & \\
\hline Adult & Adult & 505.21 & 505.76 & 502.47 & 498.91 & 4.65 \\
\hline Adult & Juvenile & 524.09 & 518.36 & 518.38 & 510.44 & 16.18 \\
\hline Adult & Total & 516.10 & 513.75 & 511.49 & 506.18 & 11.92 \\
\hline Juvenile & Adult & 502.55 & 498.00 & 499.00 & $494.26 \dagger$ & 0.00 \\
\hline Juvenile & Juvenile & 520.88 & 516.01 & 515.77 & 510.58 & 16.32 \\
\hline Juvenile & Total & 513.39 & 509.41 & 507.25 & 502.11 & 7.85 \\
\hline Total & Adult & 504.46 & 503.84 & 500.85 & 496.87 & 2.61 \\
\hline Total & Juvenile & 522.55 & 518.57 & 517.88 & 510.79 & 16.53 \\
\hline Total & Total & 514.95 & $512.04 \$$ & 510.42 & 505.75 & 11.49 \\
\hline
\end{tabular}

Notes: Candidate models included all combinations of stage-structured submodels (colonization and extinction), with and without a rescue effect, and with (SCE) and without (No SCE) a spatially correlated extinction term. Values for $\triangle$ DIC are based on a model with SCE and the inclusion of a rescue effect, as per the best-supported model, and compare the fit of stage-structured models.

$\uparrow$ The best-supported model ( $\mathrm{M}_{\text {best }}$ in the text).

$\$$ The same model as the one designated by $\dagger$, but with no stage structure and no SCE term ( $\mathrm{M}_{\text {base }}$ in the text).

Eq. 1. The important distinction is that extinction connectivity describes the distance-dependent influence of all other extinct $\left(1-P_{j, t}\right)$ populations:

$$
S_{i, t}^{e}=\sum_{j \neq i}\left(1-P_{j, t}\right) P_{j, t-1}^{*} \exp \left(-\alpha^{e} d_{i, j}\right) .
$$

Here, population size $P_{j, t-1}^{*}$ represents the magnitude of an extinction event $\left(1-P_{j, t}\right)$; i.e., larger populations going extinct have a greater influence on extinction of the focal population. The scale parameter $\alpha^{e}$ defines the correlated extinction kernel, which can be related to halving distance of the extinction pressure from extinct populations by $\log (2) / \alpha^{e} ; d_{i, j}$ is inter-patch distance. This novel approach allows the spatial scale of correlated extinction to be estimated and neighborhoods of elevated extinction risk to be identified. Extinction thus becomes a function of both local population size and connectivity to extinct colonies: $\operatorname{logit}\left(E_{i, t}\right)=\beta_{0}+\beta_{1} P_{i, t-1}^{*}$ $+\beta_{2} S_{i, t}^{e}$, where $\beta_{2}$ relates the probability of extinction to the connectivity to nearby extinction events.

We specified a hierarchy of SPOMs as follows: (1) with and without the extinction connectivity term, (2) with and without a rescue effect, and (3) using all combinations of stages in both colonization and extinction (36 candidate models; Table 1). We adopted a Bayesian approach using uninformative priors on the linear predictor scale within a biologically meaningful range (Table 2). Model discrimination was based on differences in the deviance information criteria, $\triangle \mathrm{DIC}$. Using 25000 random draws from the marginal posterior probability distributions for all parameters, we calculated the probability that a patch was occupied as predicted under the proposed model $\left(C_{i, t}\right.$ if empty at time $t-1$, and $1-E_{i, t}\left[1-C_{i, t}\right]$ if occupied at time $\left.t-1\right)$. These probabilities were used to assess model fit by comparing the predicted number of colonization and extinction events per year to the observed data. Using $\mathrm{R}$ (R Development Core Team 2011) and R2OpenBugs (Sturtz et al. 2005), models were fitted using OpenBUGS
(Lunn et al. 2009). OpenBUGS code is provided in the Supplement. Parameter estimates are presented in the text as posterior modes with $95 \%$ credible intervals $(95 \%$ CI).

\section{Results}

Patch state transitions are shown Fig. 1. On average, the annual patch occupancy of the 69 patches was $42 \%$ (range 28-58 patches). The number of extinction events per year (median 13.5, range 6-24) and colonization events per year (median 11, range 5-27) were similar, suggesting that recolonization compensated for local extinctions; i.e., the metapopulation was in apparent equilibrium. On average, 39\% (27 per year) of patches experienced turnovers (range: 22-51 per year). Median adult colony size was 2 voles (range $1-16$ ) but $41 \%$ of colonies consisted of more than a single matriline.

Juvenile dispersal was the best predictor of colonization events, whereas extinction was spatially correlated and was best explained using adult population size and including a rescue effect (Table 1). Colonization probabilities increased with proximity to extant patches and juveniles provided the best measure of connectivity (Fig. 2a, b). The scale parameter $\alpha$ was 0.0559 (95\% CI 0.0166-0.133), indicating a halving of per patch colonization pressure every $12.402 \mathrm{~km}$ (95\% CI 5.210-

TABLE 2. Posterior modal values (with Bayesian 95\% credible intervals) for parameter estimates from the best-supported parameterization of the SPOM; the priors used for all models are also given.

\begin{tabular}{lcl}
\hline \hline Parameter & Posterior mode $(95 \%$ CI $)$ & \multicolumn{1}{c}{ Prior } \\
\hline$\alpha^{e}$ & $0.78(0.31$ to 4.22$)$ & uniform $(0.02,4.5)$ \\
$\alpha$ & $0.056(0.0166$ to 0.133$)$ & uniform $(0.0001,100)$ \\
$\gamma$ & $0.37(0.28$ to 0.45$)$ & uniform $(0,100)$ \\
$\beta_{0}$ & $1.62(0.39$ to 3.40$)$ & uniform $(-100,100)$ \\
$\beta_{1}$ & $-0.75(-1.48$ to -0.41$)$ & uniform $(-100,100)$ \\
$\beta_{2}$ & $1.39(0.48$ to 4.99$)$ & uniform $(-100,100)$ \\
\hline
\end{tabular}

Note: All beta parameters are logistic regression parameters and are reported untransformed. 
- Colonization event $\quad$ o Remained occupied $\quad \square$ Extinction event $\quad \square$ Remained empty

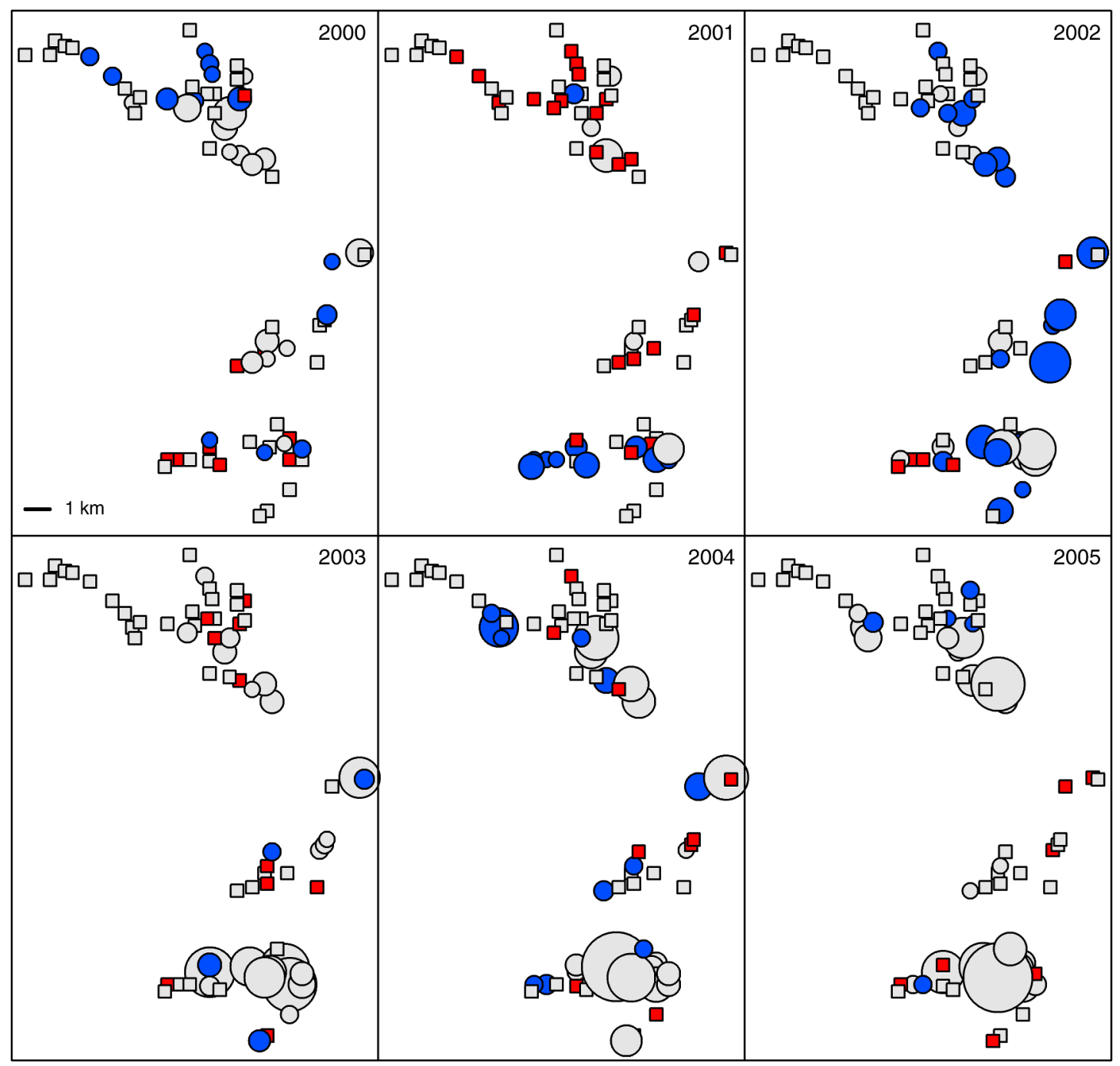

FIG. 1. Patch transition maps showing the transition states conditional on the previous year. Each symbol represents a discrete patch. Red squares denote extinction events $(1 \rightarrow 0)$, gray squares denote patches that remained empty $(0 \rightarrow 0)$, blue circles denote patches that were newly colonized $(0 \rightarrow 1)$, and gray circles denote patches that remained occupied $(1 \rightarrow 1)$. Circle size is proportional to colony size, and squares have a colony size of 0 . The distance scale is the same in all panels.

$41.857 \mathrm{~km})$. Connectivity was high; even the most distant patches (maximum inter-patch distance $28 \mathrm{~km}$ ) contributed to the colonization probability of a focal patch (Fig. 2a). Estimated per juvenile immigration rate, $\gamma$, was 0.37 (95\% CI 0.28-0.46). We also identified spatial structure in extinction risk such that extinction probabilities increased with proximity to extinct neighbors. Relative to large-scale connectivity $(\alpha=0.0559$ or $12.402 \mathrm{~km})$, the spatial scale of correlated extinctions was considerably smaller $\left(\alpha^{e}=0.78\right.$ [95\% CI $\left.0.31-4.22\right]$ or $0.89 \mathrm{~km}$ [95\% CI $0.16-2.21 \mathrm{~km}])$. Extinction pressure on a focal patch decreased with increasing distance from newly extinct colonies (Fig. 2c) and that pressure halved every $0.89 \mathrm{~km}$. The differences in spatial scales in dispersal/colonization (Fig. 2a; dashed line) and corre- lated extinctions (Fig. 2a, solid line) show two metapopulation processes operating at very different scales.

Extinction regression coefficients on the linear predictor scale were $\beta_{0}=1.62(95 \%$ CI $0.39-3.41)$ and $\beta_{1}=$ -0.75 adult $(95 \% \mathrm{CI}-1.48$ to -0.42$)$, indicating that extinction probabilities reduced with increasing numbers of established adults in a patch in the previous year (Fig. 2d). Direct comparisons of models with vs. without a rescue effect show that patch occupancy dynamics were best predicted by models that include the rescue effect (Table 1). Fig. 2c shows that, under typical conditions (median adult population size of two voles and mean colonization probability of 0.28 ), removing such a rescue effect acts to inflate the predicted extinction probability (solid vs. dash-dotted line). 

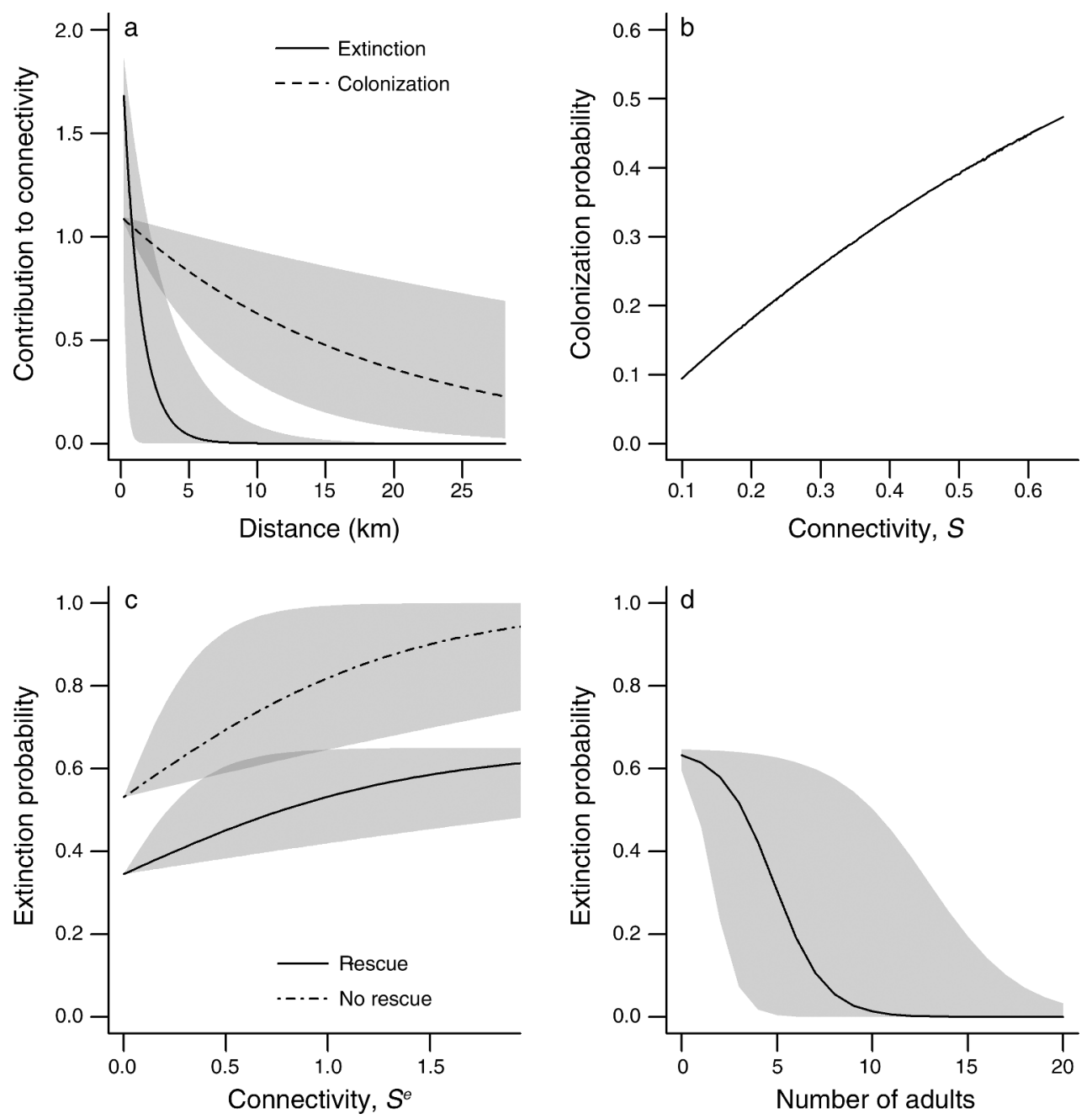

FIG. 2. Posterior modal parameter values are used for relationships in all panels. (a) Comparison of the scales of colonization and extinction using the estimated dispersal kernel (dashed line, scale parameter $\alpha$, and $N=2$ ) and the correlated extinction kernel (solid line, scale parameter $\alpha^{e}$, and $N=2$ ); shaded areas represent uncertainty in scale parameters via the $95 \%$ Bayesian credible intervals (CIs). (b) Colonization probability is an increasing function of proximity to juveniles (measured as connectivity, $S$ ), which is deterministic; hence there is no uncertainty around the line $\left(C_{i, t}=1-\exp \left[-S_{i, t}\right]\right)$. (c) The relationship, under the proposed model, between the probability that a focal patch goes extinct and proximity to newly extinct neighbors (extinction connectivity, $S^{e}$ ) for a typical scenario in which the adult population size is 2 (the median) and colonization probability is 0.28 (mean across all patches and years). The solid line shows this relationship including a rescue effect $\left(E^{*}=E[1-C]\right)$, and the shaded areas are calculated using the posterior modal values of $\beta_{0}(1.62)$ and $\beta_{1}(-0.75)$ and the $95 \%$ CIs of $\beta_{2}$ and thus denote uncertainty in $\beta_{2}$. To illustrate that removing the rescue effect increases the predicted extinction probability, the dash-dotted line shows the relationship under the same conditions with the exception that $E=E$ (i.e., no rescue effect). The shaded areas are calculated as in panel (a). (d) The relationship between extinction probability and the number of adults in the focal patch (solid line) for a typical scenario where extinction connectivity is 0.1 (mean across all patches and years) and the inclusion of a rescue effect for which colonization probability is fixed at 0.28 (mean across all patches and years). Here we do not show a comparison with and without the rescue effect. Shaded areas represent uncertainty in the intercept $\left(\beta_{0}\right)$ and the slope $\left(\beta_{1}\right)$ via the $95 \%$ CIs.

Our model predicts extinction events better than colonization events. In all but one year, the number of predicted extinctions falls within the $95 \%$ credible intervals of our predictions, whereas this is only the case for two of the six years for colonization (Fig. 3a). This result suggests that there is some important biology missing from the model that might explain the nonsystematic bias in predicted colonization among years. As a point of reference, the model just described,
$\mathrm{M}_{\text {best }}$, can be compared to the same model ignoring stage structure in colonization and extinction and excluding the extinction connectivity term: $\mathrm{M}_{\text {base }}$. We used the percentage of patches correctly classified, PCC (Freeman and Moisen 2008), to compare the ability of both models to predict individual patch occupancy states. Correct classification is achieved when the predicted occupancy probability under the proposed model is $>0.5$ for patches observed as being occupied 
and $<0.5$ for those observed as empty. The predicted probability of occupancy is $C_{i, t}$ if empty at time $t-1$, and is $1-E_{i, t}\left(1-C_{i, t}\right)$ if occupied at time $t-1$. In addition to having a lower DIC $(\triangle \mathrm{DIC}=17.78$; Table 1 : footnotes $\dagger$ vs. $\$), \mathrm{M}_{\text {best }}$ predicted individual patch occupancy states better than $\mathrm{M}_{\text {base }}$ (Fig. 3b).

\section{Discussion}

In this paper we presented a natural metapopulation of water voles that conforms to the area-isolation paradigm such that connectivity to extant populations and population size adequately describe patterns of colonization and extinction, respectively. We showed that the number of juvenile voles, rather than total population size, in the surrounding extant patches is a better predictor of colonization, whereas the number of adult voles in a patch is a better predictor of extinction events. That is, stage structure is important in metapopulation processes. We presented a novel extension of SPOMs that accounts for spatial structure in extinction using extinction connectivity, which is particularly convenient for comparing the scale of correlation in both extinction and colonization. Extinction and colonization were spatially structured but at very different scales (extinction and colonization pressure halve every $0.89 \mathrm{~km}$ and $12.40 \mathrm{~km}$, respectively; Fig. 2a). Finally, our study provided compelling evidence of a rescue effect.

This study is in agreement with many empirical studies showing that patch occupancy dynamics are better described when demographic structure is considered: in this case, stage structure rather than total population size (Crone et al. 2001, Schooley and Wiens 2005, Ozgul et al. 2009) or patch size (Pellet et al. 2007). Not considering stage structure produced biased estimates of the scale of dispersal $(\log (2) / \alpha$ : juvenile $=12.40$ $\mathrm{km}$ vs. total population $=5.52 \mathrm{~km}$ ). That juveniles should be a more informative determinant of colonization is biologically consistent with observations in water voles that most movement happens early in life (juvenile natal dispersal), as is the case for most small-mammal species (Telfer et al. 2001, Lambin et al. 2004, Le Galliard et al. 2011). It is also sensible that adult population size should determine the fate of patches in terms of their extinction risk for two reasons. First, they have near nonoverlapping breeding generations and individuals are almost never caught in their third summer; coupled with our results that juveniles are the more mobile life stage, smaller numbers of perishing adults must therefore be an informative measure of extinction risk. Secondly, colony sizes are small and, given that $59 \%$ of local populations are single family groups, natal dispersal is important to avoid inbreeding. Larger adult population sizes increase the likelihood that multiple family groups are present within a site, and we propose that the pressure to emigrate from these patches may be reduced where unrelated potential mates are present. This is seen in Fig. 2d, where patches with
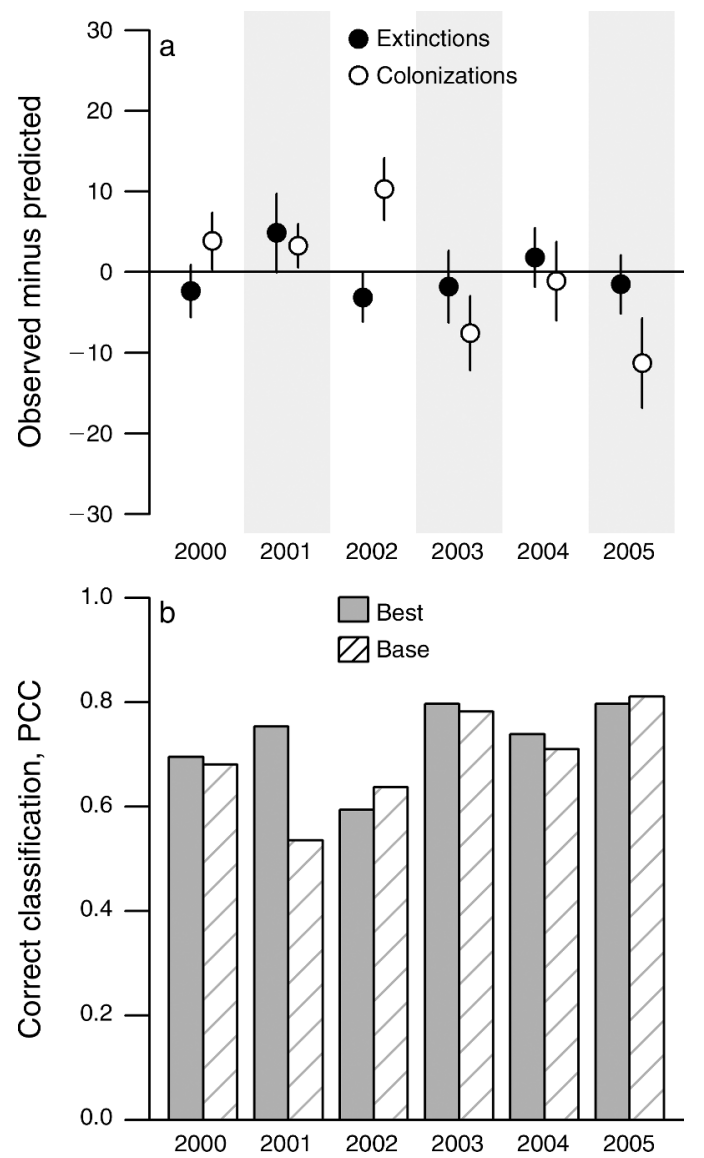

Fig. 3. (a) Difference (mean and $95 \%$ Bayesian credible interval, CI), between the observed number of turnover events and the predicted number of turnover events under the proposed $\left(\mathrm{M}_{\text {best }}\right)$ model. The figure shows, for each year, the mean of the simulated number of turnovers that were generated by summing the predicted probabilities of extinction (solid circles) and colonization (open circles) events. (b) Using the metric PCC (proportion correctly classified), we compared the predicted patch occupancy probabilities under the best-supported model $\left(\mathrm{M}_{\text {best }}\right)$ to the model with no stage structure, i.e., using total population size, and no spatially correlated extinction term $\left(\mathrm{M}_{\text {base }}\right)$. We use modal values of predicted patch occupancy probabilities from 25000 simulations under each model.

10 or more individuals have an almost zero probability of going extinct.

Using population size in SPOMs incurs added model complexity (Ovaskainen 2002). Although the ability of our model to make long-term forward predictions is limited, we show that fitting stage-structured models to patch occupancy data has important benefits for understanding the roles of demography and spatial autocorrelation in naturally occurring metapopulations. Our findings provide results that could inform sensible parameterization of predictive simulation (sensu Moilanen 2004) and integrated (Buckland et al. 2004, Harrison et al. 2011) metapopulation models. Notably, the framework presented here does not account for 
imperfect detection (Mackenzie et al. 2003). In this system, however, estimates of detection probability are high $(0.85$ CI 0.72-0.99; C. Sutherland, D. Elston, and $\mathrm{X}$. Lambin, unpublished manuscript) and a simulation study investigating the sensitivity of SPOM model parameters found that parameter estimates were not sensitive to such small detection errors (Bierman 2004).

There has been discussion as to the role of dispersal in mitigating the detrimental effect of correlated extinctions (Johst et al. 2002, Ovaskainen et al. 2002), although empirical evidence of such multi-scale processes in a natural system is rare. In their theoretical investigation of the interplay between the scales of dispersal and correlated habitat loss, Ovaskainen et al. (2002) suggest that large-scale dispersal can reduce the significance of short-scale landscape correlation. Using a natural metapopulation that appears to be in equilibrium, we have quantified the spatial scales of both dispersal (large scale, $12.40 \mathrm{~km}$ ) and correlated extinctions (small scale, $0.89 \mathrm{~km}$ ). Moreover, we show that metapopulations can, at least in the short term, persist despite correlated extinctions when dispersal distances are sufficiently large. Such short-scale correlations, although not consistent with climatic effects, could result from predator foraging behavior or disease/ pathogen transfer. Interestingly, our estimates of the scale of the correlated extinction is consistent with patterns of mustelid (Mustela nivalis or M. erminea) predation, suggested by Clinchy et al. (2002) to be affecting a similar-sized small mammal in a man-made patch network in California.

The rescue effect predicts that populations should benefit from reduced extinction risk when immigration is sufficiently frequent such that, particularly where colonies are small, their size is boosted by even a small number of immigrants (Brown and Kodric-Brown 1977). There are few empirical demonstrations of the beneficial nature of the rescue effect, and here we show convincing evidence of a nontrivial rescue effect. At median population size (two voles) and in the absence of correlated extinction, a rescue effect reduces extinction from 0.53 to 0.35 (Fig. 2c).

Our results have demonstrated that the ecologically important processes of colonization and extinction have significant dependencies on demographic stage structure. The use of stage-specific population size can better describe metapopulation processes, and we suggest that, where possible, details regarding life stage be incorporated. Demonstration of a range of processes operating within the same natural metapopulation is novel, particularly empirical quantification of the spatial scales of both colonization and extinction and the compelling evidence of a recue effect. We emphasize the need to consider spatial scale in fragmented populations beyond just distance-dependent dispersal. As predicted by theory, metapopulations can be resilient to correlated extinctions when the scale of dispersal is greater than that of extinction. While maintaining the simplicity and tractability of the SPOM, we were able to identify a variety of metapopulation processes operating at multiple spatial scales and add to the growing understanding of metapopulation processes.

\section{ACKNOWLEDGMENTS}

We thank all of the "volers and volettes" for field data collection, Chris Rix and all the staff at Inchnadamph Lodge for their hospitality and the land owners for access permission. Thanks to Stijn Bierman for the early development of the model and Justin Travis, Alex Douglas, and Olivier Cotto for comments on the manuscript. We are particularly thankful for the very detailed and helpful comments provided by two anonymous referees which helped to improve the manuscript. C. Sutherland is funded by a "Sixth Century Ph.D. Studentship" from the College of Life Sciences and Medicine of Aberdeen University, X. Lambin was supported in part by a Leverhulme Research Fellowship, and D. Elston by the Scottish Government (RESAS).

\section{Literature Cited}

Aars, J., X. Lambin, R. Denny, and A. C. Griffin. 2001. Water vole in the Scottish uplands: distribution patterns of disturbed and pristine populations ahead and behind the American mink invasion front. Animal Conservation 4:187-194.

Baguette, M., and N. Schtickzelle. 2003. Local population dynamics are important to the conservation of metapopulations in highly fragmented landscapes. Journal of Applied Ecology 40:404-412.

Bierman, S. 2004. Spatio-temporal models in animal population dynamics. Dissertation. University of Aberdeen, Aberdeen, UK.

Bjørnstad, O. N., R. A. Ims, and X. Lambin. 1999. Spatial population dynamics: analyzing patterns and processes of population synchrony. Trends in Ecology and Evolution $14: 427-432$.

Brown, J. H., and A. Kodric-Brown. 1977. Turnover rates in insular biogeography: effect of immigration on extinction. Ecology 58:445-449.

Buckland, S. T., K. B. Newman, L. Thomas, and N. B. Koesters. 2004. State-space models for the dynamics of wild animal populations. Ecological Modelling 171:157-175.

Clinchy, M., D. T. Haydon, and A. T. Smith. 2002. Pattern does not equal process: What does patch occupancy really tell us about metapopulation dynamics? American Naturalist 159:351-362.

Crone, E., D. Doak, and J. Pokki. 2001. Ecological influences on the dynamics of a field vole metapopulation. Ecology 82:831-843.

Day, J. R., and H. P. Possingham. 1995. A stochastic metapopulation model with variability in patch size and position. Theoretical Population Biology 48:333-360.

Etienne, R. S. 2000. Local populations of different sizes, mechanistic rescue effect and patch preference in the Levins metapopulation model. Bulletin of Mathematical Biology 62:943-958.

Etienne, R. S., C. J. F. ter Braak, and C. C. I. Vos. 2004. Application of stochastic patch occupancy models to real metapopulations. Pages 105-132 in I. Hanski and O. E. Gaggiotti, editors. Ecology, genetics and evolution of metapopulations. Elsevier, Burlington, Massachusetts, USA.

Freeman, E. A., and G. Moisen. 2008. PresenceAbsence: An R package for presence absence analysis. Journal of Statistical Software 23:1-31.

Gyllenberg, M., J. Hemminki, and T. Tammaru. 1999. Allee effects can both conserve and create spatial heterogeneity in population densities. Theoretical Population Biology 56:231242 . 
Hanski, I. 1991. Single-species metapopulation dynamics: concepts, models and observations. Biological Journal of the Linnean Society 42:17-38.

Hanski, I. 1994. A practical model of metapopulation dynamics. Journal of Animal Ecology 63:151-162.

Hanski, I. 1997. Predictive and practical metapopulation models: the incidence function approach. Pages 21-45 in D. Tilman and P. Kareiva, editors. Spatial ecology. Princeton University Press, Princeton, New Jersey, USA.

Hanski, I. 1998. Metapopulation dynamics. Nature 396:41-49.

Hanski, I., and O. E. Gaggiotti. 2004. Metapopulation biology: past, present, and future. Pages 3-22 in I. Hanski and O. E. Gaggiotti, editors. Ecology, genetics and evolution of metapopulations. Elsevier, Burlington, Massachusetts, USA.

Hanski, I., and O. Ovaskainen. 2000. The metapopulation capacity of a fragmented landscape. Nature 404:755-758.

Harrison, P. J., I. Hanski, and O. Ovaskainen. 2011. Bayesian state-space modeling of metapopulation dynamics in the Glanville fritillary butterfly. Ecological Monographs 81:581-598.

Heino, M., V. Kaitala, E. Ranta, and J. Lindstrom. 1997. Synchronous dynamics and rates of extinction in spatially structured populations. Proceedings of the Royal Society B 264:481-486.

Holt, A. R. 1993. Ecology at the mesoscale: the influence of regional processes on local communities. Pages 77-88 in R. E. Ricklefs and D. Schluter, editors. Species diversity in ecological communities. University of Chicago Press, Chicago, Illinois, USA.

Johst, K., R. Brandl, and S. Eber. 2002. Metapopulation persistence in dynamic landscapes: the role of dispersal distance. Oikos 98:263-270.

Kallimanis, A. S., W. E. Kunin, J. M. Halley, and S. P. Sgardelis. 2005. Metapopulation extinction risk under spatially autocorrelated disturbance. Conservation Biology 19:534-546.

Koenig, W. D. 1999. Spatial autocorrelation of ecological phenomena. Trends in Ecology and Evolution 14:22-26.

Lambin, X., J. Aars, S. B. Piertney, S. Telfer, I. Hanski, and O. E. Gaggiotti. 2004. Inferring pattern and process in small mammal metapopulations: insights from ecological and genetic data. Pages 515-540 in I. Hanski and O. E. Gaggiotti, editors. Ecology, genetics and evolution of metapopulations. Elsevier, Burlington, Massachusetts, USA.

Le Galliard, J.-F., A. Rémy, R. A. Ims, and X. Lambin. 2011. Patterns and processes of dispersal behaviour in arvicoline rodents. Molecular Ecology 21:505-523.

Lehmann, L., and N. Perrin. 2003. Inbreeding avoidance through kin recognition: choosy females boost male dispersal. American Naturalist 162:638-652.

Liebhold, A., W. D. Koenig, and O. N. Bjørnstad. 2004. Spatial synchrony in population dynamics. Annual Review of Ecology, Evolution, and Systematics 467-490.

Lunn, D., D. Spiegelhalter, A. Thomas, and N. Best. 2009. The BUGS project: Evolution, critique and future directions. Statistics in Medicine 28:3049-3067.

MacKenzie, D. I., J. D. Nichols, J. E. Hines, M. G. Knutson, and A. B. Franklin. 2003. Estimating site occupancy, colonization, and local extinction when a species is detected imperfectly. Ecology 84:2200-2207.

Matthysen, E. 2005. Density-dependent dispersal in birds and mammals. Ecography 28:403-416.

Moilanen, A. 1999. Patch occupancy models of metapopulation dynamics: efficient parameter estimation using implicit statistical inference. Ecology 80:1031-1043.

Moilanen, A. 2004. SPOMSIM: software for stochastic patch occupancy models of metapopulation dynamics. Ecological Modelling 179:533-550.

Moilanen, A., and M. Nieminen. 2002. Simple connectivity measures in spatial ecology. Ecology 83:1131-1145.

Moilanen, A., A. T. Smith, and I. Hanski. 1998. Long-term dynamics in a metapopulation of the American pika. American Naturalist 152:530-542.

Ovaskainen, O. 2002. The effective size of a metapopulation living in a heterogeneous patch network. American Naturalist 160:612-628.

Ovaskainen, O., and I. Hanski. 2003. Extinction threshold in metapopulation models. Annales Zoologici Fennici 40:81-97.

Ovaskainen, O., and I. Hanski. 2004. Metapopulation dynamics in highly fragmented landscapes. Pages $73-103$ in I. Hanski and O. E. Gaggiotti, editors. Ecology, genetics and evolution of metapopulations. Elsevier, Burlington, Massachusetts, USA.

Ovaskainen, O., K. Sato, J. Bascompte, and I. Hanski. 2002. Metapopulation models for extinction threshold in spatially correlated landscapes. Journal of Theoretical Biology 215:95-108.

Ozgul, A., M. K. Oli, K. B. Armitage, D. T. Blumstein, and D. H. Van Vuren. 2009. Influence of local demography on asymptotic and transient dynamics of a yellow-bellied marmot metapopulation. American Naturalist 173:517-530.

Pellet, J., E. Fleishman, D. S. Dobkin, A. Gander, and D. D. Murphy. 2007. An empirical evaluation of the area and isolation paradigm of metapopulation dynamics. Biological Conservation 136:483-495.

Prugh, L. R. 2009. An evaluation of patch connectivity measures. Ecological Applications 19:1300-1310.

R Development Core Team. 2011. R: A language and environment for statistical computing. R Foundation for Statistical Computing, Vienna, Austria. http://www. R-project.org

Schooley, R. L., and J. A. Wiens. 2005. Spatial ecology of cactus bugs: area constraints and patch connectivity. Ecology 86:1627-1639.

Sturtz, S., U. Ligges, and A. Gelman. 2005. R2WinBUGS: A package for running WinBUGS from R. Journal of Statistical Software 12:1-16.

Telfer, S., A. R. Holt, R. Donaldson, and X. Lambin. 2001. Metapopulation processes and persistence in remnant water vole populations. Oikos 95:31-42.

White, G. C., and K. P. Burnham. 1999. Program MARK: survival estimation from populations of marked animals. Bird Study 46:S120-S139.

\section{Supplemental Material}

\section{Appendix A}

Details of the closed-capture model that was used to estimate the probability of trapping an individual at least once (Ecological Archives E093-231-A1).

\section{Appendix B}

Photographs of the study system at different scales illustrating the extent of the study system and habitat patches (Ecological Archives E093-231-A2).

\section{Supplement}

British national grid $X Y$ coordinates of habitat patches, colony size data which give the number of individuals in a patch per year, and the OpenBUGS code for implementing the SPOM (Ecological Archives E093-231-S1). 